\title{
Tasas de crecimiento de cuatro especies nativas en plantaciones forestales comerciales en cuatro regiones de la Amazonía peruana
}

\author{
Growth rates of four native tree species in commercial plantations \\ in four regions of the Peruvian Amazon
}

Julio Ugarte-Guerra ${ }^{1,2, *}$ y Francisco Román-Dañobeytia ${ }^{3,4}$

Recibido: 16 setiembre 2020|Aceptado: 14 diciembre 2020| Publicado en línea: 18 diciembre 2020 Citación: Ugarte-Guerra, J; Román-Dañobeytia, F. 2020. Tasas de crecimiento de cuatro especies nativas en plantaciones forestales comerciales en cuatro regiones de la Amazonía peruana. Revista Forestal del Perú 35 (3, Número Especial): 44-55. DOI: http://dx.doi.org/10.21704/rfp.v35i3.1600

\begin{abstract}
Resumen
Las plantaciones forestales comerciales con especies nativas pueden proporcionar una variedad de servicios ecosistémicos, principalmente madera, carbono y recursos para la biodiversidad. Para aprovechar el potencial de producción el conocimiento de las tasas de crecimiento es fundamental para el desarrollo y promoción de plantaciones forestales comerciales en regiones tropicales. Este estudio describe la influencia de las condiciones ambientales en las tasas de crecimiento de cuatro especies forestales nativas mayormente utilizadas en plantaciones comerciales en cuatro regiones de la Amazonía peruana. Se establecieron 224 parcelas cuadradas de $400 \mathrm{~m}^{2}$ en plantaciones de diversas edades de Guazuma crinita, Calycophyllum spruceanum, Simarouba amara y Cedrelinga cateniformis. Se calculó el Incremento Medio Anual (IMA) en diámetro y altura de las cuatro especies a partir de la medición del diámetro a la altura del pecho, la altura total y la altura comercial de un total de 5974 individuos evaluados en las 224 parcelas. Las plantaciones de G. crinita y C. spruceanum fueron las que exhibieron una mayor variación en sus tasas de crecimiento, mientras que las plantaciones de $S$. amara y $C$. cateniformis fueron las que mostraron menores diferencias en sus tasas de crecimiento. Las plantaciones evaluadas en Ucayali mostraron menor crecimiento promedio. Se concluye que la variabilidad observada en las tasas de crecimiento es producto de la edad de evaluación, las características intrínsecas de la especie, así como por el historial de uso de la tierra, ya que las tasas de crecimiento fueron significativamente menores en pastizales ganaderos que en áreas agrícolas.
\end{abstract}

Palabras clave: Calycophyllum spruceanum, Cedrelinga cateniformis, Guazuma crinita, reforestación, rehabilitación, Simarouba amara

\footnotetext{
${ }^{1}$ Universidad Nacional Agraria la Molina (UNALM), Av. La Molina s/n, La Molina, Lima, Perú.

${ }^{2}$ James Cook University, Queensland, Australia.

${ }^{3}$ Programa de Desarrollo Forestal Sostenible, Inclusivo y Competitivo en la Amazonía Peruana, Servicio Nacional Fores-

tal y de Fauna Silvestre, Ministerio de Agricultura y Riego. Magdalena del Mar, Lima.

${ }^{4}$ Restaura Amazonía S.R.L. - RAMAZ. Puerto Maldonado, Madre de Dios.

* Autor de Correspondencia: julio.ugarte@gmail.com
} 


\begin{abstract}
Commercial forest plantations with native species can provide a variety of ecosystem services, mainly timber, carbon sequestration and resources for biodiversity. However, the increase of knowledge on species growth rates is key for the development and promotion of commercial forest plantations in tropical regions. This study evaluates the influence of the history of land use and abiotic factors on the growth rates of four native tree species mostly used in commercial plantations in four regions of the Peruvian Amazon. A total of 224 square plots of $400 \mathrm{~m}^{2}$ were established in plantations of different ages of the species Guazuma crinita, Calycophyllum spruceanum, Simarouba amara and Cedrelinga cateniformis. The Average Annual Increase (IMA) in diameter and height of the four species was calculated by measuring the diameter at breast height, the total height and the commercial height of a total of 5974 individuals evaluated in the 224 plots. The G. crinita and C. spruceanum plantations showed the greatest variation in growth rates, while the S. amara and C. cateniformis plantations showed the smallest differences in growth rates. The plantations evaluated in Ucayali showed lower average growth. It is concluded that the variability observed in growth rates is a product of the age of evaluation, the intrinsic characteristics of the species, as well as the history of land use, since growth rates were significantly lower in cattle pastures than in agricultural areas.
\end{abstract}

Key words: Calycophyllum spruceanum, Cedrelinga cateniformis, Guazuma crinita, reforestation, rehabilitation, Simarouba amara

\section{Introducción}

Grandes extensiones de bosque tropical se están degradando, con la consiguiente pérdida de biodiversidad y cambios climáticos globales (Barlow et al. 2016). Aunque los esfuerzos por restaurar los bosques se vienen incrementando en América Latina, estos suelen estar restringidos por la falta de financiamiento (Schweizer et al. 2018). Por otro lado, las plantaciones forestales comerciales con especies nativas se vienen estableciendo como uno de los medios para reforestar grandes áreas de paisajes deforestados o degradados, y al mismo tiempo proveen de productos maderables a la industria forestal (Griscom et al. 2017).

A medida que la oferta sostenible de madera de bosque natural decaiga y la demanda siga aumentando, la tendencia general en el futuro será hacia el aumento de la producción y la utilización de más madera cultivada en plantaciones (Guariguata et al. 2017). Varios países están interesados en mejorar los rendimientos y retornos financieros de sus plantaciones a través de la producción madera de menores dimensiones de especies de rápido crecimiento y madera de densidad media como una nueva oportunidad de mercado (Merry et al. 2009).
A pesar de que la reforestación comercial en el Perú es aún incipiente comparada con otros países de América Latina, la superficie de plantaciones se ha ido incrementando en las últimas dos décadas. Actualmente, existen más de 15300 ha de plantaciones forestales comerciales en regiones de la selva, principalmente en tierras privadas. En la Selva las especies más utilizadas han sido bolaina (Guazuma crinita), capirona (Calycophyllum spruceanum) y tornillo (Cedrelinga cateniformis), entre otras (Guariguata et al. 2017).

El fomento a la inversión pública y privada en plantaciones forestales requiere de conocimiento con respecto a los factores que influyen en las tasas de crecimiento y la productividad de las plantaciones, siendo clave la selección de especies y su establecimiento en sitios adecuados respecto a sus requerimientos ecológicos (van Breugel et al. 2011). Tanto en el Perú como en otros países de la región, este conocimiento es limitado por la falta de una red de plantaciones experimentales en diversos ecosistemas y la poca cantidad de publicaciones científicas que contribuyan a dar respaldo y seguridad a potenciales inversiones 
públicas y privadas en plantaciones forestales comerciales (Wightman et al. 2006, Guariguata et al. 2017). En este contexto, los objetivos del estudio fueron: a) Calcular las tasas de crecimiento de cuatro especies forestales nativas utilizadas en plantaciones en cuatro regiones de la Amazonía peruana; y b) Describir la influencia de factores abióticos en las tasas de crecimiento de las especies seleccionadas.

\section{Materiales y Métodos}

\section{Área de estudio}

En la región Ucayali se evaluaron plantaciones en las provincias de Padre Abad (distritos de Irazola y Von Humboldt) y Coronel Portillo (distritos de Yarinacocha y Campo Verde). En la región Huánuco el estudio comprendió las provincias de Puerto Inca (distritos de Súngaro y Codo del Pozuzo) y Leoncio Prado (distritos de Pucayacu, Rupa Rupa, José Crespo Castillo y Pueblo Nuevo). En la región Pasco se visitaron plantaciones en la provincia de Oxapampa (distrito de Palcazú) mientras que en la región San Martín el estudio abarcó las provincias de Mariscal Cáceres (distritos de Juanjui y Huicungo), Huallaga (distrito de Saposoa) y Lamas (distritos de Rumisapa, Lamas y Aucaloma) (Figura 1). Las plantaciones evaluadas en las cuatro regiones abarcaron un rango altitudinal entre los 150 y $800 \mathrm{msnm}$, y una precipitación pluvial promedio anual de entre los 1400 y 3500 mm (Cuadro 1).

\section{Especies estudiadas}

En base a consultas de documentos científicos, registros de plantaciones y consultas a expertos nacionales y locales se priorizaron cuatro especies: "bolaina" (Guazuma crinita Mart., Malvaceae), "capirona” (Calycophyllum spruceanum (Benth.) Hook. f. ex K. Schum., Rubiaceae), "marupa" (Simarouba amara Aubl., Simaroubaceae) y "tornillo" (Cedrelinga cateniformis (Ducke) Ducke, Fabaceae). En las cuatro regiones se encontraron mayormente plantaciones de bolaina y marupa; plantaciones de capirona se hallaron sólo en las regiones de Ucayali, Huánuco y San Martín, mientras que plantaciones de tornillo sólo se localizaron en
Huánuco y Pasco. Las especies seleccionadas tienen una amplia distribución geográfica en el trópico americano y abarcan una variedad de familias y características ecológicas que pueden ayudar a interpretar su desempeño en las plantaciones evaluadas (Cuadro 2).

\section{Evaluación de plantaciones}

Se establecieron 224 parcelas cuadradas de $400 \mathrm{~m}^{2}(20 \times 20 \mathrm{~m})$ en plantaciones forestales comerciales de las especies seleccionadas que tuvieran entre 2 y 20 años de edad (Cuadro 1). Se midieron el diámetro a la altura del pecho (DAP), la altura total y la altura comercial de un total de 5974 individuos en las 224 parcelas. El DAP se midió con cinta diamétrica, mientras que las alturas total y comercial fueron medidas con un clinómetro. A partir de entrevistas con los gerentes de las plantaciones se recabó información a sobre el historial de uso de la tierra previo a la plantación, edad de la plantación, y reconocimiento visual de áreas con mayor y menor productividad dentro de las plantaciones.

En cada una de las parcelas se hicieron 3 perforaciones en el suelo de $31 \mathrm{~cm}^{3}$ cada una con tubos de muestreo de suelos de acero inoxidable, para formar una muestra compuesta, que fue dispuesta en bolsas ziploc y enviada para su análisis al Laboratorio de Suelos y Fertilizantes de la Universidad Nacional Agraria La Molina. A cada una de las muestras se les practicó el análisis de caracterización que incluyó cálculos de $\mathrm{pH}$, conductividad eléctrica (CE), materia orgánica $(\mathrm{MO})$, fósforo $(\mathrm{P})$, potasio $(\mathrm{K})$, arena, limo, arcilla, capacidad de intercambio catiónico (CIC), cationes cambiables, suma de cationes, suma de bases y porcentaje de saturación de bases.

\section{Análisis de datos}

Se calculó el Incremento Medio Anual (IMA), tanto en diámetro como en altura, para cada uno de los individuos evaluados en las parcelas, a partir de la siguiente fórmula:

$$
\mathrm{IMA}=(\mathrm{Hf}-\mathrm{Hi}) / \mathrm{T}
$$

Donde: $\mathrm{Hf}=$ Diámetro $(\mathrm{cm})$ o altura total (m) al momento de la evaluación; Hi = Diámetro $(\mathrm{cm})$ o altura total $(\mathrm{m})$ al instalar la 


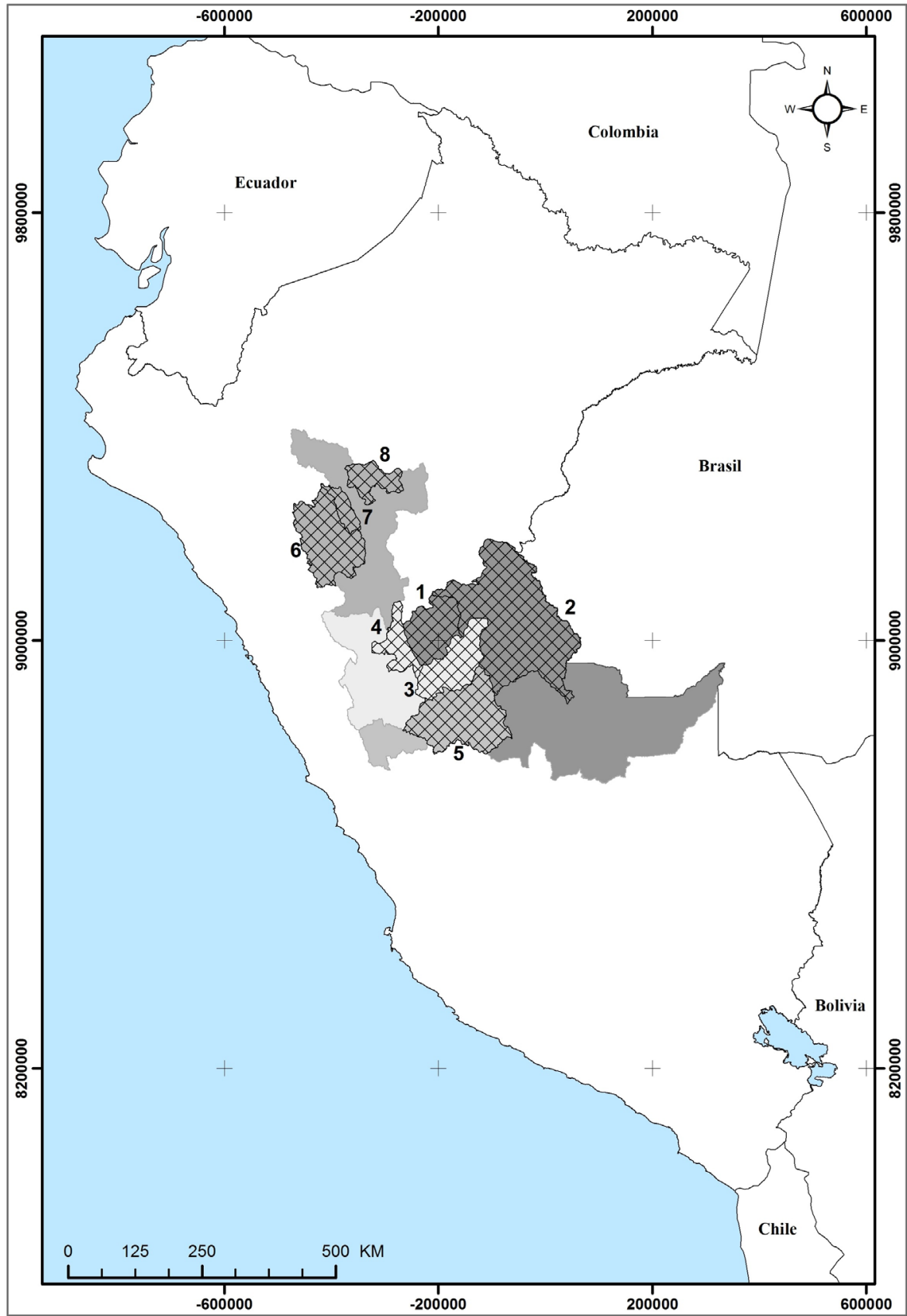

Figura 1. Mapa de ubicación mostrando las ocho provincias dentro de las cuatro regiones de la Amazonía peruana donde se realizó el estudio. Provincias de Padre Abad (1), Coronel Portillo (2), Puerto Inca (3), Leoncio Prado (4), Oxapampa (5), Mariscal Cáceres (6), Huallaga (7) y Lamas (8). 


\begin{tabular}{|l|c|c|c|c|}
\hline Características & Ucayali & Huánuco & Pasco & San Martín \\
\hline Altitud (msnm) & $150-288$ & $204-771$ & $309-409$ & $258-790$ \\
\hline Precipitación anual (mm)* & 2344 & 3472 & 3429 & 1478 \\
\hline Edad (años) & $1-18$ & $2-62$ & $4-22$ & $2-14$ \\
\hline Número de parcelas evaluadas (ind.) & $56(1688)$ & $65(1560)$ & $56(1162)$ & $47(1564)$ \\
$\bullet \quad$ Guazuma crinita & $16(556)$ & $21(545)$ & $16(251)$ & $15(503)$ \\
$\bullet \quad$ Calycophyllum spruceanum & $20(757)$ & $13(225)$ & 0 & $25(910)$ \\
- Simarouba amara & $20(375)$ & $20(721)$ & $20(748)$ & $7(151)$ \\
Cedrelinga cateniformis & 0 & $4(69)$ & $20(163)$ & 0 \\
\hline Edad en años & $1-18$ & $2-62$ & $4-22$ & $2-14$ \\
- Guazuma crinita & $1-17$ & $2-8$ & 7 & $2-5$ \\
Calycophyllum spruceanum & $2-18$ & $3-25$ & - & $2-7$ \\
- Cimarouba amara $\quad$ Cedrelinga cateniformis & 9 & $3-4$ & $4-5$ & $2-14$ \\
\hline
\end{tabular}

* Elaborado con base en Senamhi (2018).

Cuadro 1. Características abióticas y número de parcelas evaluadas en plantaciones comerciales de cuatro especies en cuatro regiones de la Amazonía peruana.

\begin{tabular}{|c|c|c|c|c|}
\hline Nombre científico & Nombre común & Familia & Distribución & $\begin{array}{c}\text { Gremio ecológico y } \\
\text { hábitat }\end{array}$ \\
\hline Guazuma crinita & Bolaina blanca (PER) & Malvaceae & $\begin{array}{c}\text { Oeste de } \\
\text { Sudamérica y } \\
\text { Brasil. }\end{array}$ & $\begin{array}{c}\text { Intolerante a la sombra, } \\
\text { vegetación secundaria } \\
\text { temprana. }\end{array}$ \\
\hline $\begin{array}{l}\text { Calycophyllum } \\
\text { spruceanum }\end{array}$ & $\begin{array}{l}\text { Capirona (PER), } \\
\text { Pau-Mulato (BRA), } \\
\text { Guayabete (COL). }\end{array}$ & Rubiaceae & $\begin{array}{c}\text { Oeste de } \\
\text { Sudamérica y } \\
\text { Brasil. }\end{array}$ & $\begin{array}{l}\text { Intolerante a la sombra, } \\
\text { vegetación secundaria. }\end{array}$ \\
\hline Simarouba amara & $\begin{array}{l}\text { Marupa (PER, COL, } \\
\text { BRA), Capulli (ECU), } \\
\text { Chiriuana (VEN). }\end{array}$ & Simaroubaceae & América tropical & $\begin{array}{l}\text { Tolerante a la sombra, } \\
\text { vegetación secundaria. }\end{array}$ \\
\hline $\begin{array}{l}\text { Cedrelinga } \\
\text { cateniformis }\end{array}$ & $\begin{array}{l}\text { Tornillo (PER), } \\
\text { Cedrorana (BRA), } \\
\text { Seique (ECU). }\end{array}$ & Fabaceae & $\begin{array}{c}\text { Oeste de } \\
\text { Sudamérica y } \\
\text { Brasil. }\end{array}$ & $\begin{array}{l}\text { Tolerante a la sombra, } \\
\text { vegetación secundaria. }\end{array}$ \\
\hline
\end{tabular}

Elaborado con base en ITTO (2018).

Cuadro 2. Características de las especies evaluadas en plantaciones forestales comerciales en cuatro regiones de la Amazonía peruana.

plantación; $\mathrm{T}$ = Tiempo transcurrido (en años) entre la instalación de la plantación y el momento de la evaluación.

A los datos calculados del IMA se les aplicó la prueba de Shapiro-Wilk, para evaluar la normalidad de los residuos de las variables de respuesta, y además se realizaron transformaciones logarítmicas en el caso del IMA en altura y diámetro para satisfacer los supuestos de normalidad y homogeneidad de varianzas (Fry 1993, Scheiner y Gurevitch 2001). Se realizaron análisis de varianza (ANOVA) para evaluar diferencias significativas en el IMA en diámetro y altura de cada especie entre las cuatro regiones estudiadas. Posteriormente se utilizó la Prueba de $t$ para evaluar diferencias significativas en el IMA en diámetro y altura, así como en las propiedades del suelo, para determinar la influencia del historial de uso previo de la tierra en las variables de crecimiento.

\section{Resultados}

\section{Tasas de crecimiento por regiones}

Se detectaron diferencias estadísticamente significativas en el IMA en altura y diámetro 


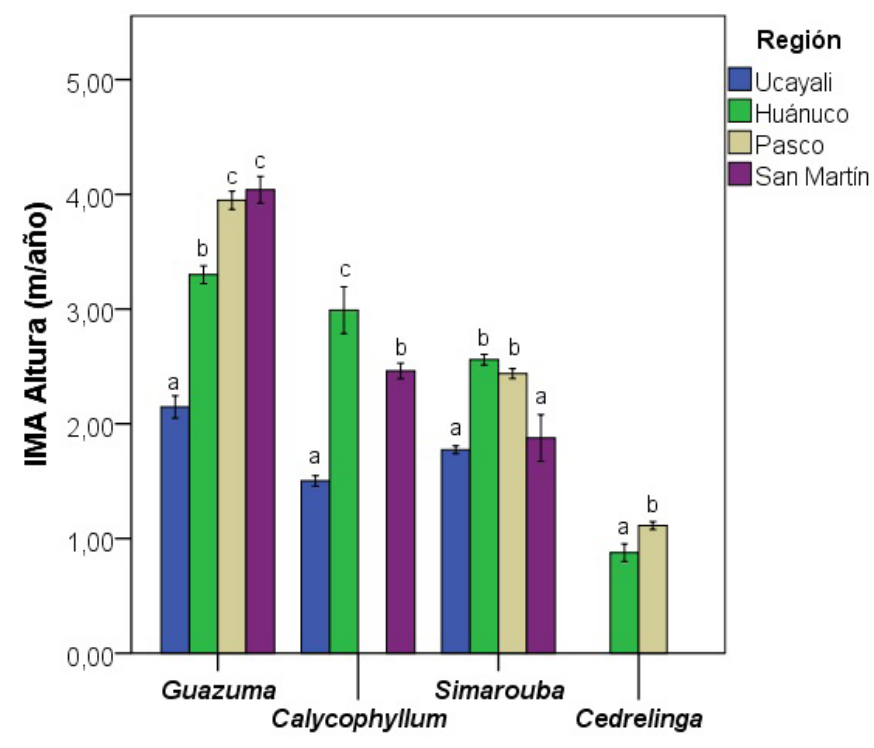

Figura 2. Diferencias en el Incremento Medio Anual (IMA) en altura de cuatro especies forestales en cuatro regiones de la Amazonía peruana. Letras diferentes encima de las barras de error revelan diferencias estadísticamente significativas (ANOVA, Prueba de Tukey, $P<0.05$ ).

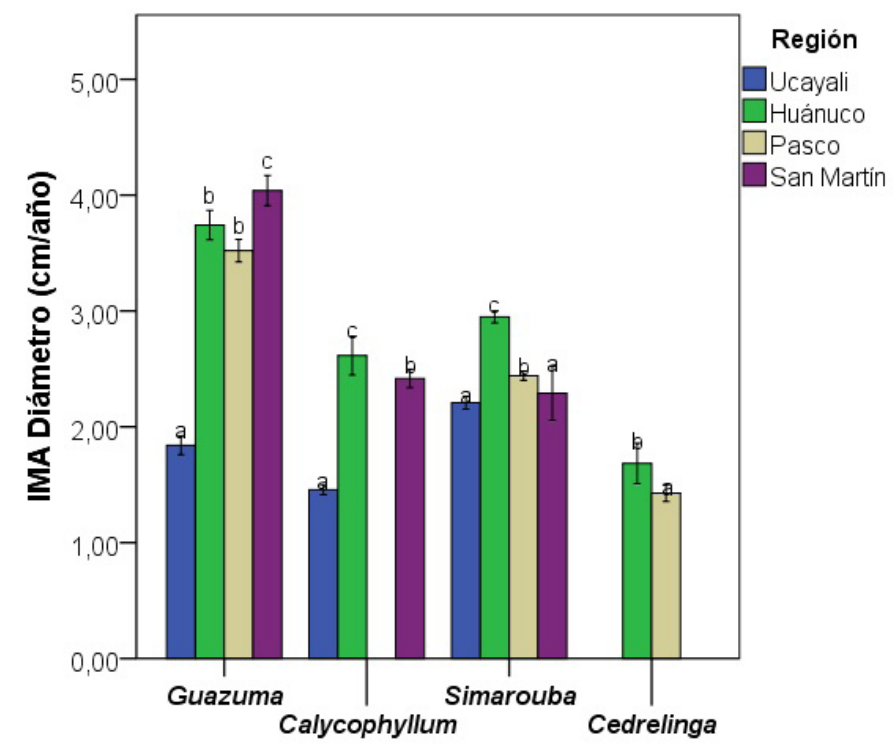

Figura 3. Diferencias en el Incremento Medio Anual (IMA) en diámetro de cuatro especies forestales en cuatro regiones de la Amazonía peruana. Letras diferentes encima de las barras de error revelan diferencias estadísticamente significativas (ANOVA, Prueba de Tukey, $P<0.05$ ). 
de las especies estudiadas en cada una de las regiones. Las mayores diferencias fueron encontradas en las plantaciones de Guazuma crinita y Calycophyllum spruceanum, especialmente entre Ucayali y San Martín para el caso de Guazuma crinita, mientras que para Calycophyllum spruceanum la mayor variación fue observada entre las plantaciones de Ucayali y Huánuco (Fig. 2).

Guazuma crinita y Calycophyllum spruceanum. El IMA en altura de las plantaciones de Guazuma crinita fue $48 \%$ y $36 \%$ mayor en San Martín y Huánuco, con respecto al IMA promedio registrado Ucayali (Fig. 2). Asimismo, el IMA en diámetro fue $54 \%$ mayor en San Martín y $49 \%$ mayor en las regiones de Pasco y Huánuco, con respecto a las plantaciones de Guazuma crinita en Ucayali (Fig, 3). En el caso de Calycophyllum spruceanum, el IMA en altura tuvo una variación del $50 \%$ y el $40 \%$, mientras que el IMA en diámetro fue $44 \%$ y $39 \%$ mayor en las plantaciones de Huánuco y San Martín con respecto a las de Ucayali (Figs. 2 y 3$)$.
Simarouba amara y Cedrelinga cateniformis. Las plantaciones de Simarouba amara y $\mathrm{Ce}$ drelinga cateniformis fueron las que mostraron menores diferencias entre las cuatro regiones estudiadas. En el caso de Simarouba amara, se registró que el IMA en altura fue mayor en las regiones de Pasco y Huánuco, mientras que el IMA en diámetro fue mayor en Huánuco que el IMA promedio de Simarouba amara registrado en Ucayali y San Martín (Figs. 2 y 3). En cuanto a las plantaciones de Cedrelinga cateniformis, se observó que el IMA en altura fue mayor en Pasco y el IMA de diámetro fue mayor en Huánuco (Figs. 2 y 3 ).

\section{Influencia del historial de uso en las tasas de crecimiento}

El historial de uso de la tierra previo a la instalación de las plantaciones demostró tener una fuerte influencia en las tasas de crecimiento, tanto en altura como en diámetro. En las cuatro regiones, se evidenciaron mayores tasas de crecimiento en áreas que fueron usadas para agricultura en comparación con áreas de historial ganadero. En cuanto al IMA en altu-

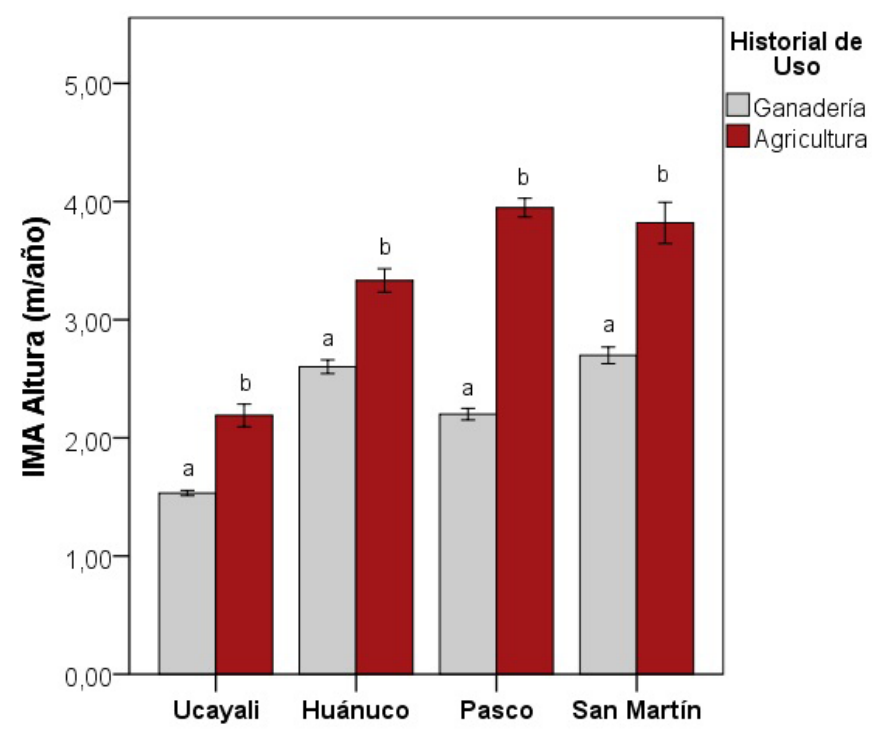

Figura 4. Diferencias en el Incremento Medio Anual (IMA) en altura en 4 regiones de la Amazonía peruana por influencia del historial de uso previo de la tierra. Letras diferentes encima de las barras de error revelan diferencias estadísticamente significativas (Prueba de $\mathrm{t}, P<0.05$ ). 


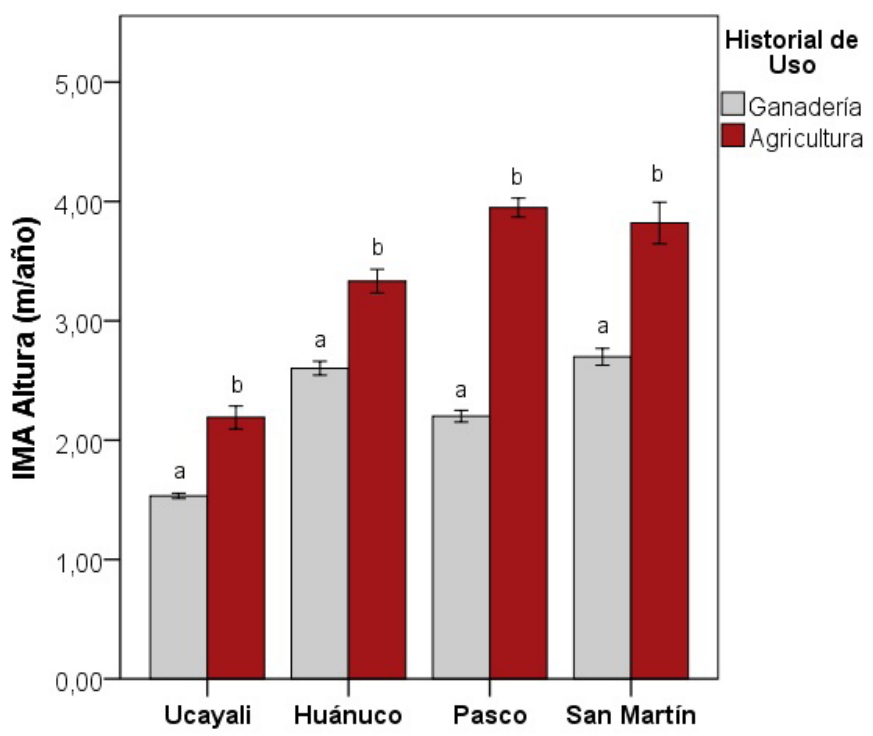

Figura 5. Diferencias en el Incremento Medio Anual (IMA) en diámetro en 4 regiones de la Amazonía peruana por influencia del historial de uso previo de la tierra. Letras diferentes encima de las barras de error revelan diferencias estadísticamente significativas (Prueba de t, $P<0.05$ ).

ra, los mayores incrementos promedio para todas las especies se encuentran en las tierras con antecedentes de uso agrícola en la región Pasco, mientras que en Ucayali se apreciaron los menores crecimientos tanto con antecedentes agrícolas como de uso ganadero (Fig. 4). En el caso del IMA en diámetro, la mayor variación por efecto del historial de uso fue encontrada en las regiones de Pasco y San Martín (34\% y $20 \%$ respectivamente), mientras que las menores diferencias fueron observadas en las regiones de Ucayali y Huánuco $(11 \%$ y $7 \%$, respectivamente) (Fig. 5).

\section{Propiedades del suelo}

Se registraron diferencias significativas en 16 de las 17 propiedades del suelo estudiadas en plantaciones con historial de uso ganadero y agrícola (Cuadro 3). La comparación muestra que los promedios de 13 de las 17 variables edáficas estudiadas ( $\mathrm{pH}, \mathrm{CE}, \mathrm{MO}, \mathrm{P}, \mathrm{K}$, Arena, Arcilla, $\mathrm{CIC}, \mathrm{Ca}^{+2}, \mathrm{~K}^{+}$, suma de cationes, suma de bases y \% Sat. bases) son significativamente mayores en plantaciones establecidas en áreas usadas previamente para agricultura que en áreas de ganadería. Sólo en tres de las variables de suelo analizadas (limo, $\mathrm{Mg}^{+2}$ y $\mathrm{Al}^{+3}+\mathrm{H}^{+}$) los promedios son mayores en plantaciones establecidas en áreas de ganadería que en plantaciones con historial de uso agrícola (Cuadro 3).

\section{Discusión}

Este estudio presenta los resultados de una evaluación extensiva de plantaciones forestales comerciales nunca antes realizada ni publicada en un medio de divulgación científica. Esta evaluación incluyó la evaluación de casi 6000 árboles en 224 parcelas de $400 \mathrm{~m}^{2}$ distribuidas en 17 distritos y 8 provincias de 4 regiones de la Amazonía peruana. Si bien consideramos que este esfuerzo constituye una muestra representativa del universo de plantaciones forestales comerciales con especies nativas en la Amazonía peruana, los resultados de este estudio deben tomarse con cautela. Particularmente en la región Ucayali, que fue la región con las tasas de crecimiento más bajas, podrían existir algunas zonas no evaluadas cercanas a Aguaytía que podrían ser mucho más productivas para plantaciones, especialmente de Guazuma que reporta valores de hasta $8.6 \mathrm{~cm}$ promedio de 


\begin{tabular}{|c|c|c|c|c|}
\hline \multirow{2}{*}{\multicolumn{2}{|c|}{ Propiedades del suelo }} & \multicolumn{2}{|c|}{ Historial de uso } & \multirow{2}{*}{$\begin{array}{c}\text { Nivel de } \\
\text { significancia }^{1}\end{array}$} \\
\hline & & Ganadería & Agricultura & \\
\hline \multicolumn{2}{|c|}{$\mathrm{pH}(1: 1)$} & $5.06 \pm 1.30$ & $5.45 \pm 1.29$ & $* *$ \\
\hline \multicolumn{2}{|c|}{ C.E. $(\mathrm{dS} / \mathrm{m})$} & $0.15 \pm 0.11$ & $0.21 \pm 0.14$ & $* *$ \\
\hline \multicolumn{2}{|c|}{ M.O. (\%) } & $3.28 \pm 1.64$ & $3.41 \pm 1.50$ & $* *$ \\
\hline \multicolumn{2}{|c|}{$\mathrm{P}(\mathrm{ppm})$} & $4.88 \pm 5.21$ & $5.49 \pm 5.68$ & $* *$ \\
\hline \multicolumn{2}{|c|}{$\mathrm{K}(\mathrm{ppm})$} & $115.96 \pm 106.81$ & $180.09 \pm 180.71$ & $* *$ \\
\hline \multicolumn{2}{|c|}{ Arena (\%) } & $43.44 \pm 14.32$ & $46.82 \pm 13.43$ & $* *$ \\
\hline \multicolumn{2}{|c|}{ Limo (\%) } & $33.21 \pm 9.66$ & $27.27 \pm 8.03$ & $* *$ \\
\hline \multicolumn{2}{|c|}{ Arcilla (\%) } & $24.32 \pm 11.60$ & $25.90 \pm 9.59$ & $* *$ \\
\hline \multirow{6}{*}{$\begin{array}{l}\text { Cationes } \\
\text { cambiables }\end{array}$} & $\mathrm{CIC}(\mathrm{meq} / 100 \mathrm{~g})$ & $17.15 \pm 12.99$ & $18.59 \pm 11.12$ & $* *$ \\
\hline & $\mathrm{Ca}^{+2}$ & $9.96 \pm 11.78$ & $12.15 \pm 11.36$ & $* *$ \\
\hline & $\mathrm{Mg}^{+2}$ & $2.52 \pm 3.54$ & $2.17 \pm 1.65$ & $* *$ \\
\hline & $\mathrm{K}^{+}$ & $0.33 \pm 0.26$ & $0.51 \pm 0.50$ & $* *$ \\
\hline & $\mathrm{Na}^{+}$ & $0.17 \pm 0.10$ & $0.17 \pm 0.12$ & $\mathrm{~ns}$ \\
\hline & $\mathrm{Al}^{+3}+\mathrm{H}^{+}$ & $0.88 \pm 1.13$ & $0.51 \pm 0.77$ & $* *$ \\
\hline \multicolumn{2}{|c|}{ Suma de cationes } & $13.85 \pm 14.33$ & $15.50 \pm 12.58$ & $* *$ \\
\hline \multicolumn{2}{|c|}{ Suma de bases } & $12.97 \pm 14.93$ & $14.99 \pm 13.04$ & $* *$ \\
\hline \multicolumn{2}{|c|}{$\%$ Saturación de bases } & $59.10 \pm 36.55$ & $68.34 \pm 32.76$ & $* *$ \\
\hline
\end{tabular}

${ }^{1} \mathrm{~ns}$ : diferencias no significantivas; $* \mathrm{p}<0.05 ; * * \mathrm{p}<0.01$.

Cuadro 3. Comparación de propiedades físico-químicas del suelo en plantaciones establecidas en áreas con historial de uso ganadero y agrícola.

diámetro a los $10 \mathrm{~cm}$ del suelo en parcelas de 12 meses de edad en zonas aluviales (Rochon et al. 2007).

Las tasas de crecimiento registradas en las plantaciones de Guazuma crinita permiten estimar un turno final de corte entre los 6 y 8 años en las regiones de Huánuco, Pasco y San Martín (Cuadro 4), mientras que para las plantaciones de Guazuma crinita evaluadas en Ucayali el turno estimado asciende a los 14 años. Igualmente, en el caso de Calycophyllum spruceanum las tasas de crecimiento permiten estimar un turno de corte final de entre 15 y 18 años en las regiones de Huánuco y San Martín, mientras que en Ucayali las bajas tasas de crecimiento hacen que el tiempo de espera para el corte final exceda los 25 años (Cuadro 4). En el caso de Simarouba amara y Cedrelinga cateniformis, las tasas de crecimiento no han tenido una variación tan importante entre las regiones como en las dos especies anteriores, estimándose un turno final de corta de entre 14 y 18 años para Simarouba amara, y de entre 30 y 35 años para Cedrelinga cateniformis (Cuadro 4).

La alta variabilidad observada en las tasas de crecimiento de Guazuma crinita y Calycophyllum spruceanum, así como la relativamente poca variación en Simarouba amara y Cedrelinga cateniformis, puede deberse al estatus sucesional de las especies y a la influencia de la calidad de sitio (Rozendaal et al. 2006). Otros estudios han demostrado que especies sucesionales iniciales, intolerantes a la sombra (ej. Guazuma crinita y Calycophyllum spruceanum para este estudio) suelen crecer más rápido y asignar más biomasa a la parte aérea cuando los recursos son abundantes, y crecer lentamente y asignar más biomasa a las raíces en niveles bajos de disponibilidad de recursos (Román-Dañobeytia et al. 2012, Santiago et al. 


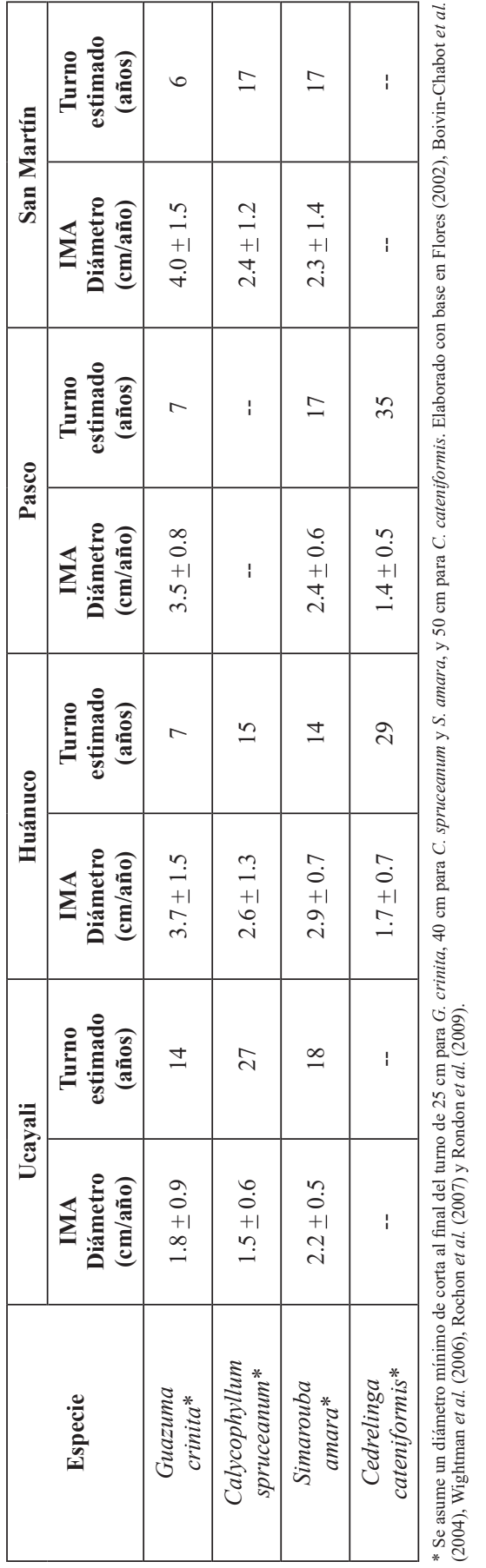

2012). Evidencia en la morfología de las plantas refleja que este tipo de especies presentan raíces superficiales y extensas, que han sido asociadas con un rol más efectivo en la exploración y explotación de zonas con alta disponibilidad de nutrientes (Paz 2003, Martínez-Garza et al. 2013a); mientras que especies tolerantes a la sombra, de sucesión avanzada (ej. Simarouba amara y Cedrelinga cateniformis para este estudio) suelen tener raíces profundas y una menor capacidad para convertir recursos abundantes en mayor crecimiento y biomasa (Russo et al. 2005, Krause et al. 2012).

Nuestro estudio demuestra que las tasas de crecimiento son significativamente menores en pastizales ganaderos que en áreas agrícolas. Estos resultados se explican también por las diferencias significativas en la mayoría de propiedades del suelo analizadas entre estas dos categorías de historia de uso. Varios estudios confirman que la ausencia de cobertura arbórea, el pisoteo constante del ganado en suelos arcillosos (por lo general durante décadas) y la elevada precipitación en regiones tropicales húmedas, propician una mayor degradación del suelo en los pastizales ganaderos en términos de compactación, pérdida de nutrientes y de materia orgánica entre otros, que dificultan la sobrevivencia y crecimiento de la vegetación arbórea natural o inducida (Braz et al. 2013, Martínez-Garza et al. 2013b). Por otro lado, la agricultura de subsistencia en la Amazonía suele ocupar la tierra por menor tiempo en comparación con la ganadería, y si bien hay también importantes cambios en cuanto a pérdida de nutrientes, las características estructurales del suelo no se degradan tanto como en el caso de la ganadería, permitiendo una recuperación de la vegetación más rápida y diversa (Fearnside y Barbosa 1998, Sohng et al. 2017).

\section{Conclusiones}

Los datos analizados corroboran que el crecimiento de Guazuma crinita y Calycophyllum spruceanum es sensible a la variación en el historial de uso previo de la tierra y la calidad de sitio, mientras que Simarouba amara y Cedrelinga cateniformis son especies cuyas 
tasas de crecimiento presentaron una menor variación entre las regiones estudiadas. Los resultados describen la importancia de la selección del sitio forestal para orientar plantaciones forestales comerciales en la Amazonía peruana hacia áreas de mayor productividad, principalmente aquellas con antecedentes de producción agrícola. Para una mayor precisión se debe intensificar el muestreo en áreas que pueden tener mayor fertilidad natural como las áreas aluviales. Una adecuada selección del sitio en función de los antecedentes y datos de precipitación y fertilidad es determinante para seleccionar la especie a instalar que justifique la inversión en plantaciones.

\section{Agradecimientos}

Este estudio fue posible gracias al financiamiento del Programa de Desarrollo Forestal Sostenible, Inclusivo y Competitivo en la Amazonía Peruana (SERFOR-CAF) a través del servicio Tasas de crecimiento en especies forestales prioritarias para el establecimiento de plantaciones con fines comerciales en 04 regiones de la Amazonia peruana (Orden de servicio 0001020). Se agradece el invaluable apoyo de Mg. France Cabanillas e Ings. Jhon Farfan e Ignacio Piqueras durante el trabajo de campo y el análisis de la información. Se agradece también la colaboración del Ing. Jorge Chávez de Bosques Amazónicos en Ucayali, Bach. Russel Robles y Bach. Lismendi Yedinela en Huánuco, Ing. Rober Medina en Pasco, e Ingenieros Daniel Angulo, Dick Vergara y Tito Vergara en San Martin. Finalmente, se agradece a todas las personas naturales y empresas que facilitaron el ingreso a sus predios y dieron las facilidades para la realización del trabajo de campo.

\section{Bibliografía}

Barlow, J; Lennox, D; Ferreira, J; Berenguer, E; Lees, AC; Mac Nally, R; Thomson, JR; de Barros Ferraz, SF; Louzada, J; Oliveira, VHF; Parry, L; de Castro Solar, RR; Vieira, ICG; Aragão, L; Begotti, RA; Braga, RF; Cardoso, TM; de Oliveira Junior, RC; Souza Junior, CM; Moura, NG; Nunes, SS; Siqueira, JV; Pardini, R; Silveira, JM;
Vaz-de-Mello, FZ; Veiga, RCS; Venturieri, A; Gardner, T. 2016. Anthropogenic disturbance in tropical forests can double biodiversity loss from deforestation. Nature 535:144-159.

Boivin-Chabot, S; Margolis, HA; Weber, JC. 2004. Variation in coppice-shoot growth among provenances of Calycophyllum spruceanum Benth. in the Peruvian Amazon Basin. Forest Ecology and Management 198:249-260.

Braz, AM; Fernadez, AR; Alleoni, LRF. 2013. Soil attributes after the conversion from forest to pasture in amazon. Land Degradation \& Development 24(1):33-38.

Fearnside, PM; Barbosa, RI. 1998. Soil carbon changes from conversion of forest to pasture in Brazilian Amazonia. Forest Ecology and Management 108:147-166.

Flores, Y. 2002. Crecimiento y productividad de plantaciones de seis especies forestales nativas de 20 años de edad en el Bosque Alexander von Humboldt, Amazonia Peruana. Tesis Mg. Sc. Turrialba, Costa Rica, CATIE. 86 p.

Fry, JC. 1993. Biological data analysis: a practical approach. Oxford, Reino Unido, Oxford University Press. 418 p.

Griscom, BW; Adams, J; Ellis, PW; Houghton, RA; Lomax, G; Miteva,DA; Schlesinger, WH; Shoch, D; Siikamäki, JV; Smith, P; Woodbury, P; Zganjar, C; Blackman, A; Campari, J; Conant, RT; Delgado, C; Elias, P; Gopalakrishna, T; Hamsik, MR; Herrero, M; Kiesecker, J; Landis, E; Laestadius, L; Leavitt, SM; Minnemeyer, S; Polasky, S; Potapov, P; Putz, FE; Sanderman, J; Silvius, M; Wollenberg, E; Fargione, J. 2017. Natural climate solutions. PNAS 114(44):11645-11650.

Guariguata, MR; Arce, J; Ammour, T; Capella, JL. 2017. Las plantaciones forestales en Perú: reflexiones, estatus actual y perspectivas a futuro. Bogor, Indonesia, CIFOR. 40 p. (Documento Ocasional n. ${ }^{\circ} 169$ ).

ITTO (International Tropical Timber Organization, Japón). 2018. [Buscar especies] (en línea, sitio web). Consultado 2 feb. 2019. Disponible en http://www.tropicaltimber.info/. 
Krause, GH; Winter, K; Matsubara, S. 2012. Photosynthesis, photoprotection, and growth of shade-tolerant tropical tree seedlings under full sunlight. Photosynthesis Research 113:273-285. DOI: https://doi.org/10.1007/s11120-012-9731-z.

Martínez-Garza, C; Bongers, F; Poorter, L. 2013a. Are functional traits good predictors of species performance in restoration plantings in tropical abandoned pastures?. Forest Ecology and Management 303:35-45.

Martínez-Garza, C; Tobon, W; Campo, J; Howe, HF. 2013b. Drought mortality of tree seedlings in an eroded tropical pasture. Land Degradation \& Development 24(3):287-295.

Merry, F; Soares-Filho, B; Nepstad, D; Amacher, G; Rodrigues, H. 2009. Balancing Conservation and Economic Sustainability: The Future of the Amazon Timber Industry. Environmental Management 44:395-407. DOI: https://doi. org/10.1007/s00267-009-9337-1.

Paz, H. 2003. Root/shoot allocation and root architecture in seedlings: variation among forest sites, microhabitats and ecological groups. Biotropica 35(3):318-332.

Rochon, C; Margolis, HA; Weber, JC. 2007. Genetic variation in growth of Guazuma crinita (Mart.) trees at an early age in the Peruvian Amazon. Forest Ecology and Management 243:291-298.

Román-Dañobeytia, FJ; Levy-Tacher, SI; Aronson, J; Ribeiro-Rodrigues, R; CastellanosAlbores, J. 2012. Testing the performance of fourteen native tropical tree species in two abandoned pastures of the Lacandon Rainforest Region of Chiapas, Mexico. Restoration Ecology 20(3):378-386.

Rondon, XJ; Gorchov, DL; Noble Junior, RB. 2009. Projection of tree growth and timber volume following strip clear-cutting in the Peruvian Amazon. Forest Ecology and Management 257(2):588-599.

Rozendaal, DMA; Hurtado, VH; Poorter, L. 2006. Plasticity in leaf traits of 38 tropical tree species in response to light; relationships with light demand and adult stature. Functional Ecology 20:207-216.
Russo, SE; Davies, SJ; King, DA; Tan, S. 2005. Soil-related performance variation and distributions of tree species in a Bornean rain forest. Journal of Ecology 93(5):879-889.

Santiago, LS; Wright, S; Harms, KE; Yavitt, JB; Korine, C; Garcia, MN; Turner, BL. 2012. Tropical tree seedling growth responses to nitrogen, phosphorus and potassium addition. Journal of Ecology 100(2):309-316.

Scheiner, S; Gurevitch, J. 2001. Design and Analysis of Ecological Experiments. 2 ed. Nueva York, Estados Unidos de América, Oxford University Press. 432 p.

Schweizer, D; Meli, P; Brancalion, P; Guariguata, M. 2018. Oportunidades y desafíos para la gobernanza de la restauración del paisaje forestal en América Latina. Bogor, Indonesia, CIFOR. 72 p. (Documentos Ocasionales n. ${ }^{\circ} 182$ ).

Senamhi (Servicio Nacional de Meteorología e Hidrología del Perú). 2018. Datos Hidrometeorológicos a nivel nacional (en línea, sitio web). Consultado 18 ene. 2019. Disponible en https://www.senamhi.gob.pe/?p=estaciones.

Sohng, J; Singhakumara, BMP; Ashton, MS. 2017. Effects on soil chemistry of tropical deforestation for agriculture and subsequent reforestation with special reference to changes in carbon and nitrogen. Forest Ecology and Management 389:331-340.

van Breugel, M; Hall, JS; Craven, DJ; Gregoire, TG; Park, A; Dent, DH; Wishnie, MH; Mariscal, E; Deago, J; Ibarra, D; Cedeño, N; Ashton, MS. 2011. Early growth and survival of 49 tropical tree species across sites differing in soil fertility and rainfall in Panama. Forest Ecology and Management 261(10):1580-1589.

Wightman, KE; Cornelius, JP; Ugarte-Guerra, LJ. 2006. ¡Plantemos madera!: manual sobre el establecimiento, manejo y aprovechamiento de plantaciones maderables para productores de la Amazonía peruana. Lima, Perú, ICRAF. 194 p. (Manual Técnico n. ${ }^{\circ}$ ). 